\title{
THE GROWTH RATE OF AN ENTIRE FUNCTION AND THE HAUSDORFF DIMENSION OF ITS JULIA SET
}

\author{
WALTER BERGWEILER, BOGUSŁAWA KARPIŃSKA, AND GWYNETH M. STALLARD
}

\begin{abstract}
Let $f$ be a transcendental entire function in the Eremenko-Lyubich class $B$. We give a lower bound for the Hausdorff dimension of the Julia set of $f$ that depends on the growth of $f$. This estimate is best possible and is obtained by proving a more general result concerning the size of the escaping set of a function with a logarithmic tract.
\end{abstract}

\section{INTRODUCTION AND MAIN RESULT}

Let $f$ be a transcendental entire function and denote by $f^{n}, n \in \mathbb{N}$, the $n$th iterate of $f$. The Fatou set, $F(f)$, is defined to be the set of points, $z \in \mathbb{C}$, such that $\left(f^{n}\right)_{n \in \mathbb{N}}$ forms a normal family in some neighbourhood of $z$. The complement, $J(f)$, of $F(f)$ is called the Julia set of $f$. An introduction to the basic properties of these sets can be found in, for example, [5].

The Hausdorff dimension of the Julia set of an entire function $f$ was first considered by McMullen [14] who proved that $\operatorname{dim} J(f)=2$ if $f(z)=\lambda e^{z}$, where $\lambda \in \mathbb{C} \backslash\{0\}$. Taniguchi [24] extended this result to functions $f$ of the form

$$
f(z)=\int_{0}^{z} P(t) e^{Q(t)} d t+c,
$$

where $P$ and $Q$ are polynomials and $c \in \mathbb{C}$.

There is a close relationship between the Julia set and the escaping set

$$
I(f)=\left\{z: f^{n}(z) \rightarrow \infty \text { as } n \rightarrow \infty\right\}
$$

which was first studied for a general transcendental entire function $f$ by Eremenko [8]. Among other results, Eremenko proved that the Julia set is always equal to the boundary of the escaping set.

An important role in complex dynamics is played by the Eremenko-Lyubich class $B$ consisting of all transcendental entire functions for which the set of critical values and finite asymptotic values is bounded. This class contains the functions considered by McMullen and Taniguchi mentioned above. Eremenko and Lyubich [9] proved that, if $f \in B$, then $I(f) \subset J(f)$. Thus, for such functions, a lower bound for the size of the Julia set can be obtained by estimating the size of the escaping set. An alternative method for obtaining a lower bound for the size of the Julia set of a function in the class $B$ is given in [3].

1991 Mathematics Subject Classification. 37F10 (primary), 30D05, 30D15 (secondary).

All three authors were supported by the EU Research Training Network CODY. The first author was also supported by the G.I.F., the German-Israeli Foundation for Scientific Research and Development, Grant G-809-234.6/2003 and the ESF Research Networking Programme HCAA. The second author was also supported by Polish MNiSW Grant N N201 023433 and PW Grant 504G 11200011 000. The latter grant supported a visit of the first and third authors to Warsaw, during which most of the work for this paper was carried out. 
The goal of this paper is to relate the Hausdorff dimension of the Julia set of a function in the class $B$ to the growth rate of the function. Recall that the order of an entire function $f$ is defined by

$$
\rho(f)=\limsup _{r \rightarrow \infty} \frac{\log \log M(r, f)}{\log r},
$$

where $M(r, f)=\max _{|z|=r}|f(z)|$. An entire function $f$ has finite order if and only if there exists $\rho(f) \in[0, \infty)$ such that, for each $\varepsilon>0$, there exists $r_{\varepsilon}>0$ such that

$$
|f(z)| \leq \exp \left(|z|^{\rho(f)+\varepsilon}\right) \text { for }|z|>r_{\varepsilon} \text {. }
$$

Note that, if $f$ is of the form (1.1), then the order of $f$ is equal to the degree of $Q$.

Barański [2] and Schubert [20] proved that $\operatorname{dim} J(f)=2$ for any function $f$ of finite order in the class $B$. The hypothesis that $f$ has finite order cannot be omitted since it is known [21] that, for each $\varepsilon>0$, there exists a function $f \in B$ for which $\operatorname{dim} J(f)<1+\varepsilon$. In fact, for each $d \in(1,2)$ there exists a function $f \in B$ for which $\operatorname{dim} J(f)=d$; see [23]. On the other hand, it was shown in $[22$ that $\operatorname{dim} J(f)>1$ for any $f \in B$.

We now state the main result of the paper. We note that the examples in [23] show that this estimate of $\operatorname{dim} J(f)$ is best possible.

Theorem 1.1. Let $f$ be an entire function in the class $B$ and let $q \geq 1$. Suppose that, for each $\varepsilon>0$, there exists $r_{\varepsilon}>0$ such that

$$
|f(z)| \leq \exp \left(\exp \left((\log |z|)^{q+\varepsilon}\right)\right) \quad \text { for }|z| \geq r_{\varepsilon} .
$$

Then

$$
\operatorname{dim} J(f) \geq 1+\frac{1}{q}
$$

Our result shows that $\operatorname{dim} J(f)=2$ for $f \in B$ not only if $f$ has finite order, but more generally if

$$
\limsup _{r \rightarrow \infty} \frac{\log \log \log M(r, f)}{\log \log r}=1 .
$$

It thus strengthens the results of Barański [2] and Schubert [20]. Examples of functions in the class $B$ can be constructed, for example, by using contour integrals; see [15], [21] and [23]. This technique yields examples of functions $f \in B$ which have infinite order but satisfy (1.3). For any $q>1$, examples of functions $f \in B$ which have infinite order and satisfy (1.2) were constructed in [19]. These examples have the additional property that all the path-connected components of $J(f)$ are points.

Next we note that, if $f \in B$, then $\rho(f) \geq \frac{1}{2}$. This observation seems to have appeared first in [13], [6]; see also [18, Lemma 3.5]. This implies that a function $f \in B$ cannot satisfy (1.2) for some $q<1$.

Finally we note that the hypothesis (1.2) can also be written in the form

$$
\limsup _{r \rightarrow \infty} \frac{\log \log \log M(r, f)}{\log \log r} \leq q \text {. }
$$

Such limits or, more generally, limits of the form

$$
\limsup _{r \rightarrow \infty} \frac{\log ^{i+k} M(r, f)}{\log ^{k} r},
$$

for certain $i, k \geq 0$, have been considered, for example, in [11, Chapter IV].

The main tool used by Eremenko and Lyubich to study a function $f$ in the class $B$ was a logarithmic change of variable. Choose $R>|f(0)|$ such that $\Delta_{R}=\{z \in \mathbb{C}:|z|>R\}$ 
contains no critical values and no asymptotic values of $f$. Then every component $D$ of $f^{-1}\left(\Delta_{R}\right)$ is simply connected and $f: D \rightarrow \Delta_{R}$ is a universal covering. Now let $H=\{z \in \mathbb{C}: \operatorname{Re} z>\log R\}$. The map exp :H $\rightarrow \Delta_{R}$ is also a universal covering and so there exists a biholomorphic map $G: D \rightarrow H$ such that $f=\exp \circ G$. We define $F: \exp ^{-1}(D) \rightarrow H$ by $F(z)=G\left(e^{z}\right)$ so that $\exp F(z)=f\left(e^{z}\right)$. We say that $F$ is the function obtained from $f$ by a logarithmic change of variable.

In many applications of this method it is irrelevant how $f$ behaves outside $D$, or whether $f$ is even defined outside $D$. This leads to the following definition.

Definition 1.1. Let $D \subset \mathbb{C}$ be an unbounded domain in $\mathbb{C}$ whose boundary consists of piecewise smooth curves. Suppose that the complement of $D$ is unbounded. Let $f$ be a complex-valued function whose domain of definition contains the closure $\bar{D}$ of $D$. Then $D$ is called a logarithmic tract of $f$ if $f$ is holomorphic in $D$ and continuous in $\bar{D}$ and if there exists $R>0$ such that $f: D \rightarrow \Delta_{R}$ is a universal covering.

If $D$ is a logarithmic tract of $f$, then

$$
I(f, D)=\left\{z \in D: f^{n}(z) \in D \text { for all } n \in \mathbb{N} \text { and } f^{n}(z) \rightarrow \infty \text { as } n \rightarrow \infty\right\} .
$$

In fact it was shown in [16. Theorem 2.4] that $I(f, D)$ always has at least one unbounded component; see also [7] for a generalisation to the case when $D$ is a direct tract.

Note that, if $f \in B$, then $f$ has a logarithmic tract $D$. Clearly $I(f, D) \subset I(f)$ and so $I(f, D) \subset J(f)$ by the result of Eremenko and Lyubich [9] mentioned earlier. Hence Theorem 1.1 follows from the following general result.

Theorem 1.2. Let $f$ be a function with a logarithmic tract D. Suppose that, for each $\varepsilon>0$, there exists $r_{\varepsilon}>0$ such that (1.2) holds for $z \in D$. Then $\operatorname{dim} I(f, D) \geq 1+1 / q$.

In order to prove Theorem 1.2 , we work with the function $F: \exp ^{-1}(D) \rightarrow H$ obtained from $f$ by a logarithmic change of variable. For each $p>q-1$, we construct a set $E_{p} \subset \exp ^{-1}(D)$ such that

$$
\operatorname{Re} F^{n}(z) \rightarrow \infty \text { for } z \in E_{p}
$$

and

$$
\operatorname{dim} E_{p} \geq 1+\frac{1}{1+p}
$$

We note that $\exp \left(E_{p}\right) \subset I(f, D)$. Since $E_{p}$ and $\exp \left(E_{p}\right)$ have the same Hausdorff dimension and $p$ can be chosen to be arbitrarily close to $q-1$, this is sufficient to prove Theorem 1.2 .

To construct the sets $E_{p}$, we use a generalisation of the method used by McMullen in [14]. As in [14], the sets $\exp E_{p}$ that we construct consist of points that 'zip to infinity'; that is, they belong to the set

$$
Z(f, D)=\left\{z \in I(f, D): \frac{1}{n} \log \log \left|f^{n}(z)\right| \rightarrow \infty \text { as } n \rightarrow \infty\right\} .
$$

In our situation, however, more sophisticated arguments are needed to construct such points. The machinery required for this construction is set up in Section 3 where we introduce the notion of an 'admissible square' - a square where $F$ grows regularly in a certain sense. The sets $E_{p}$ consist of points whose forward iterates all lie in an admissible square. We estimate the dimensions of the sets $E_{p}$ in Section 4 . Our calculations are based on ideas similar to those used by McMullen in [14] - again, more delicate arguments are needed as we do not have uniform bounds on the quantities involved. 
We note that in contrast to Barański [2] and Schubert [20] we do not use Ahlfors' distortion theorem.

\section{Preliminary Lemmas}

We begin with the following lemma about real functions. It is very similar to [4, Lemma 3], but we include the proof for completeness.

Lemma 2.1. Let $\alpha, \beta:[c, \infty) \rightarrow \mathbb{R}$ be continuous and increasing. Suppose that $\beta$ is differentiable, that $\alpha$ is absolutely continuous, that $\alpha(x) \leq \beta(x)$, that $\lim _{x \rightarrow \infty} \beta(x)=\infty$ and that $\beta^{\prime}(x)>0$. Define $\psi:[\beta(c), \infty) \rightarrow(0, \infty)$ by $\psi(t)=\beta^{\prime}\left(\beta^{-1}(t)\right)=1 /\left(\beta^{-1}\right)^{\prime}(t)$. If $K>1$, then

$$
\alpha^{\prime}(x) \leq K \psi(\alpha(x))
$$

on a set of $x$-values of lower density at least $(K-1) / K$.

Of course, the inequality (2.1) makes sense only for values of $x$ where $\alpha$ is differentiable, but absolutely continuous functions are differentiable almost everywhere. Thus, if $L_{K}$ denotes the set where (2.1) holds, then the points where $\alpha$ is not differentiable are in the complement of $L_{K}$.

Proof. For $y>c$ we define

$$
C_{y}=\left\{x \in[c, y]: \alpha^{\prime}(x)>K \psi(\alpha(x))\right\} .
$$

Then

$$
\begin{aligned}
K \int_{C_{y}} d x & \leq \int_{C_{y}} \frac{\alpha^{\prime}(x)}{\psi(\alpha(x))} d x \\
& \leq \int_{c}^{y} \frac{\alpha^{\prime}(x)}{\psi(\alpha(x))} d x \\
& =\int_{\alpha(c)}^{\alpha(y)} \frac{d u}{\psi(u)} \\
& =\beta^{-1}(\alpha(y))-\beta^{-1}(\alpha(c)) \\
& \leq y-\beta^{-1}(\alpha(c)),
\end{aligned}
$$

and we deduce that the set of $x$-values where $\alpha^{\prime}(x)>K \psi(\alpha(x))$ has upper density at most $1 / K$. The conclusion follows.

We next recall the following classical result. Inequalities (2.2) and (2.3) are Koebe's distortion theorem and (2.4) is Koebe's $\frac{1}{4}$-theorem. Here, and throughout the paper, $B(a, r)$ denotes the open disk around $a$ of radius $r$.

Lemma 2.2. Let $g: B(a, r) \rightarrow \mathbb{C}$ be univalent, $\rho \in(0,1)$ and $z \in B(a, \rho r)$. Then

$$
\begin{gathered}
\frac{\rho}{(1+\rho)^{2}}\left|g^{\prime}(a)\right| r \leq|g(z)-g(a)| \leq \frac{\rho}{(1-\rho)^{2}}\left|g^{\prime}(a)\right| r, \\
\frac{1-\rho}{(1+\rho)^{3}}\left|g^{\prime}(a)\right| \leq\left|g^{\prime}(z)\right| \leq \frac{1+\rho}{(1-\rho)^{3}}\left|g^{\prime}(a)\right|
\end{gathered}
$$

and

$$
g(B(a, r)) \supset B\left(g(a), \frac{1}{4}\left|g^{\prime}(a)\right| r\right) .
$$


Remark. If $\rho=\frac{1}{2}$ then (2.3) takes the form

$$
\frac{4}{27}\left|g^{\prime}(a)\right| \leq\left|g^{\prime}(z)\right| \leq 12\left|g^{\prime}(a)\right|
$$

and so, if $z, w \in B\left(a, \frac{1}{2} r\right)$,

$$
\frac{1}{81} \leq\left|\frac{g^{\prime}(z)}{g^{\prime}(w)}\right| \leq 81
$$

The following result is a simple consequence of Koebe's distortion theorem.

Lemma 2.3. Let $g: B(a, r) \rightarrow \mathbb{C}$ be univalent, $\rho \in\left(0, \frac{1}{2}\right)$ and $z, w \in B(a, \rho r)$. Then

$$
\left|g(z)-g(w)-g^{\prime}(a)(z-w)\right| \leq 26\left|g^{\prime}(a)\right| \rho|z-w| .
$$

Proof. It follows from (2.3) that if $\zeta \in B\left(a, \frac{1}{2} r\right)$, then

$$
\left|g^{\prime}(\zeta)-g^{\prime}(a)\right| \leq\left|g^{\prime}(\zeta)\right|+\left|g^{\prime}(a)\right| \leq 13\left|g^{\prime}(a)\right| \text {. }
$$

Schwarz's lemma yields

$$
\left|g^{\prime}(\zeta)-g^{\prime}(a)\right| \leq 26\left|g^{\prime}(a)\right| \frac{|\zeta-a|}{r}
$$

for $\zeta \in B\left(a, \frac{1}{2} r\right)$. Hence

$$
\left|g(z)-g(w)-g^{\prime}(a)(z-w)\right|=\left|\int_{w}^{z}\left(g^{\prime}(\zeta)-g^{\prime}(a)\right) d \zeta\right| \leq 26\left|g^{\prime}(a)\right| \rho|z-w|,
$$

for $z, w \in B(a, \rho r)$.

We shall also need the following version of Vitali's lemma [10, Lemma 4.8].

Lemma 2.4. Let $\left\{B\left(x_{i}, r_{i}\right): i \in I\right\}$ be a collection of balls in $\mathbb{R}^{n}$ whose union is bounded. Then there exists a finite subset $E$ of $I$ such that $B\left(x_{i}, r_{i}\right) \cap B\left(x_{j}, r_{j}\right)=\emptyset$ for $i, j \in E$, $i \neq j$, and

$$
\bigcup_{i \in I} B\left(x_{i}, r_{i}\right) \subset \bigcup_{i \in E} B\left(x_{i}, 4 r_{i}\right)
$$

\section{Admissible squares}

Let $f$ and $D$ be as in Theorem 1.2. We may assume that $R=1$ in the definition of the logarithmic tract and that $0 \notin D$. Let $H=\{z \in \mathbb{C}: \operatorname{Re} z>0\}$ be the right half-plane and let $F: \exp ^{-1}(D) \rightarrow H$ be the function obtained from $f$ by a logarithmic change of variable, as described in Section 1 . Note that $F$ is $2 \pi i$-periodic and the restriction of $F$ to a component of $\exp ^{-1}(D)$ maps this component bijectively onto $H$.

We now fix $\varepsilon>0$ and $p>q-1+\varepsilon$. Recall that we are aiming to construct a set $E_{p} \subset \exp ^{-1}(D)$ such that

$$
\operatorname{Re} F^{n}(z) \rightarrow \infty \text { for } z \in E_{p}
$$

In order to do this, we let $x_{0}=\inf \left\{\operatorname{Re} z: z \in \exp ^{-1}(D)\right\}$ and consider the function $h:\left(x_{0}, \infty\right) \rightarrow(0, \infty)$ defined by

$$
h(x)=\max _{y \in \mathbb{R}} \operatorname{Re} F(x+i y) .
$$

Note that $h$ is increasing by the maximum principle. Moreover, $h$ is convex by analogy to Hadamard's three circles theorem. Thus $h$ has left and right derivatives at all points. If $z_{x}=x+i y_{x}$ is a point such that $h(x)=\operatorname{Re} F\left(z_{x}\right)$, then $F^{\prime}\left(z_{x}\right)$ is real and lies between 
the left and right derivative of $h$ at $x$. Except for the countable set $C$ where $h$ is not differentiable, we thus have

$$
h^{\prime}(x)=F^{\prime}\left(z_{x}\right)
$$

We now obtain estimates for the size of $h$ and $h^{\prime}$.

Lemma 3.1. Let $h:\left(x_{0}, \infty\right) \rightarrow(0, \infty)$ and the countable set $C$ be defined as above. Then there exists $x_{\varepsilon} \geq x_{0}$ and a set $L \subset\left(x_{0}, \infty\right) \backslash C$ of density 1 such that

$$
\begin{gathered}
h(x) \leq \exp \left(x^{q+\varepsilon}\right) \quad \text { for } x \in\left(x_{\varepsilon}, \infty\right), \\
\frac{h^{\prime}(x)}{h(x)} \leq x^{p} \quad \text { for } x \in L, \\
\frac{h^{\prime}(x)}{h(x)} \geq \frac{1}{4 \pi} \quad \text { for } x \in\left(x_{0}, \infty\right) \backslash C, \\
h(x) \geq \exp \left(\frac{1}{13} x\right) \quad \text { for } x \in\left(x_{\varepsilon}, \infty\right),
\end{gathered}
$$

and

$$
h^{\prime}(x) \geq \exp \left(\frac{1}{14} x\right) \quad \text { for } x \in\left(x_{\varepsilon}, \infty\right) \backslash C .
$$

Proof. The upper bound (3.2) for $h$ follows directly from hypothesis (1.2).

To obtain an estimate for $h^{\prime}$ we note that it follows from (3.2) that we can apply Lemma 2.1 with $\alpha(x)=h(x)$ and $\beta(x)=\exp \left(x^{q+\varepsilon}\right)$. We have $\beta^{-1}(t)=(\log t)^{1 /(q+\varepsilon)}$, $\beta^{\prime}(x)=(q+\varepsilon) \beta(x) x^{q+\varepsilon-1}$ and

$$
\psi(t)=(q+\varepsilon) t(\log t)^{\frac{q+\varepsilon-1}{q+\varepsilon}}
$$

so that

$$
h^{\prime}(x) \leq K(q+\varepsilon) h(x)(\log h(x))^{\frac{q+\varepsilon-1}{q+\varepsilon}} \leq K(q+\varepsilon) h(x) x^{q+\varepsilon-1}
$$

on a set of lower density at least $(K-1) / K$. Since $p>q-1+\varepsilon$, the right hand side of (3.7) is smaller than $h(x) x^{p}$ for large $x$, if $K>1$ is fixed. The upper bound (3.3) for $h^{\prime} / h$ now follows.

Now recall that $z_{x}=x+i y_{x}$ is a point such that $h(x)=\operatorname{Re} F\left(z_{x}\right)$. It follows from Koebe's $\frac{1}{4}$-theorem (2.4) and from (3.1) that if $\varphi$ is the branch of $F^{-1}$ that maps $F\left(z_{x}\right)$ to $z_{x}$, then $\varphi\left(B\left(F\left(z_{x}\right), h(x)\right)\right.$ contains a disk around $z_{x}$ of radius $r$, where

$$
r=\frac{h(x) \varphi^{\prime}\left(F\left(z_{x}\right)\right)}{4}=\frac{h(x)}{4 F^{\prime}\left(z_{x}\right)}=\frac{h(x)}{4 h^{\prime}(x)} \quad \text { for } x \in\left(x_{0}, \infty\right) \backslash C .
$$

On the other hand, $\varphi\left(B\left(F\left(z_{x}\right), h(x)\right) \subset \exp ^{-1}(D)\right.$ and $\exp ^{-1}(D)$ does not contain disks of radius greater than $\pi$. The lower bound (3.4) for $h^{\prime} / h$ now follows. Integrating (3.4) and noting that $4 \pi<13$, we obtain (3.5). The lower bound (3.6) for $h^{\prime}$ follows from (3.4) and (3.5).

We are now in a position to define the key idea of an admissible square. 
Definition 3.1. For $z \in \mathbb{C}$ and $r>0$ we consider the square

$$
S(z, r)=\{\zeta \in \mathbb{C}:|\operatorname{Re} \zeta-\operatorname{Re} z| \leq r,|\operatorname{Im} \zeta-\operatorname{Im} z| \leq r\} .
$$

We call $z$ the centre of $S(z, r)$. We say that $S(z, r)$ is admissible if $100<r<\frac{1}{2} \operatorname{Re} z$ and

$$
\text { length }([\operatorname{Re} z-r, \operatorname{Re} z+r] \cap L) \geq \frac{7}{4} r,
$$

where length(.) denotes the one-dimensional Lebesgue measure and $L$ is the set of density 1 from (3.3).

The following result is the main tool that we use in the construction of the set $E_{p}$.

Lemma 3.2. Given $\tau>1$, there exist positive constants $c_{0}, c_{1}, c_{2}, c_{3}$ with the following properties:

If $S(z, r)$ is an admissible square and $x=\operatorname{Re} z>c_{0}$, then there exist $m \in \mathbb{N}$ with $m>$ $c_{3} r x^{p}$, compact subsets $A_{1}, A_{2}, \ldots, A_{m}$ of $S\left(z, \frac{1}{4} r\right)$ and points $a_{1}, a_{2}, \ldots, a_{m}$ in $S\left(z, \frac{1}{4} r\right)$ such that $F$ maps $A_{j}$ bijectively onto an admissible square centred at $F\left(a_{j}\right)$,

$$
\begin{gathered}
B\left(a_{j}, \frac{c_{1}}{x^{p}}\right) \subset A_{j} \subset B\left(a_{j}, \frac{c_{2}}{x^{p}}\right) \subset B\left(a_{j}, 1\right) \subset S\left(z, \frac{1}{4} r\right), \\
B\left(a_{j}, \frac{\tau c_{2}}{x^{p}}\right) \subset\left\{\zeta \in \mathbb{C}:|\operatorname{Re} \zeta-\operatorname{Re} z| \leq \frac{1}{4} r,|\operatorname{Im} \zeta-\operatorname{Im} z| \leq \pi+1\right\}
\end{gathered}
$$

and

$$
\operatorname{Re} F\left(a_{j}\right) \geq \exp \left(\frac{1}{15} x\right) \geq c_{0}
$$

for $j=1,2, \ldots, m$. Moreover,

$$
\operatorname{Re} a_{j+1}>\operatorname{Re} a_{j}+\frac{\tau c_{2}}{x^{p}}
$$

for $j=1,2, \ldots, m-1$.

Proof. Let

$$
L^{\prime}=\left[x-\frac{1}{4} r+1, x+\frac{1}{4} r-1\right] \cap L .
$$

Since $S(z, r)$ is admissible we have

$$
\text { length }\left(L^{\prime}\right) \geq \frac{1}{4} r-2 \geq \frac{1}{5} r .
$$

We apply Lemma 2.4 to the intervals

$$
\left(u-3 \frac{h(u)}{h^{\prime}(u)}, u+3 \frac{h(u)}{h^{\prime}(u)}\right), \quad u \in L^{\prime} .
$$

We obtain $u_{1}, u_{2}, \ldots, u_{n} \in L^{\prime}$ such that if we put

$$
r_{k}=\frac{h\left(u_{k}\right)}{h^{\prime}\left(u_{k}\right)}
$$

then

$$
\left(u_{j}-3 r_{j}, u_{j}+3 r_{j}\right) \cap\left(u_{k}-3 r_{k}, u_{k}+3 r_{k}\right)=\emptyset \quad \text { for } j \neq k,
$$

and

$$
L^{\prime} \subset \bigcup_{k=1}^{n}\left(u_{k}-12 r_{k}, u_{k}+12 r_{k}\right)
$$


It follows that

$$
\sum_{k=1}^{n} r_{k} \geq \frac{1}{24} \operatorname{length}\left(L^{\prime}\right) \geq \frac{1}{120} r .
$$

Now let $w_{k}=z_{u_{k}}$; that is, $\operatorname{Re} w_{k}=u_{k}$ and $h\left(u_{k}\right)=\operatorname{Re} F\left(w_{k}\right)$. Since $F$ is $2 \pi i$-periodic, we may choose $w_{k}$ such that

$$
\left|\operatorname{Im} w_{k}-\operatorname{Im} z\right| \leq \pi
$$

We denote by $\varphi_{k}$ the branch of the inverse function of $F$ for which $\varphi_{k}\left(F\left(w_{k}\right)\right)=w_{k}$. Then

$$
\varphi_{k}^{\prime}\left(F\left(w_{k}\right)\right)=\frac{1}{F^{\prime}\left(w_{k}\right)}=\frac{1}{h^{\prime}\left(u_{k}\right)}
$$

by (3.1) so that

$$
h\left(u_{k}\right) \varphi_{k}^{\prime}\left(F\left(w_{k}\right)\right)=r_{k} .
$$

Let

$$
W_{k}=\varphi_{k}\left(S\left(F\left(w_{k}\right), \frac{1}{4} h\left(u_{k}\right)\right)\right) .
$$

Since $\varphi_{k}$ is univalent in the right half-plane $H$ and

$$
B\left(F\left(w_{k}\right), \frac{1}{4} h\left(u_{k}\right)\right) \subset S\left(F\left(w_{k}\right), \frac{1}{4} h\left(u_{k}\right)\right) \subset B\left(F\left(w_{k}\right), \frac{1}{2} h\left(u_{k}\right)\right),
$$

we deduce from Koebe's distortion theorem (2.2) and (3.18) that

$$
B\left(w_{k}, \frac{4}{25} r_{k}\right) \subset W_{k} \subset B\left(w_{k}, 2 r_{k}\right) .
$$

Now let $\delta$ be a small positive number to be fixed later. We put

$$
m_{k}=\left[\delta r_{k} x^{p}\right] \quad \text { and } \quad \rho_{k}=\frac{h^{\prime}\left(u_{k}\right)}{x^{p}} .
$$

Note that if $0 \leq l \leq m_{k}$ and $\delta<\frac{1}{3}$, then, for large $x$,

$$
l \delta \rho_{k}+\delta^{2} \rho_{k} \leq m_{k} \delta \rho_{k}+\delta^{2} \rho_{k} \leq 2 \delta^{2} r_{k} x^{p} \rho_{k}=2 \delta^{2} h\left(u_{k}\right)<\frac{1}{4} h\left(u_{k}\right) .
$$

For $0 \leq l \leq m_{k}$ we now define

and

$$
\begin{gathered}
v_{k, l}=\varphi_{k}\left(F\left(w_{k}\right)+l \delta \rho_{k}\right), \\
S_{k, l}=S\left(F\left(w_{k}\right)+l \delta \rho_{k}, \delta^{2} \rho_{k}\right), \\
V_{k, l}=\varphi_{k}\left(S_{k, l}\right)
\end{gathered}
$$

$$
J_{k, l}=\left\{u \in \mathbb{R}:\left|u-\left(h\left(u_{k}\right)+l \delta \rho_{k}\right)\right| \leq \delta^{2} \rho_{k}\right\} .
$$

The interval $J_{k, l}$ is thus the projection of $S_{k, l}$ onto the real axis. If $\delta$ is sufficiently small, the intervals $J_{k, l}$ are pairwise disjoint, and so the same holds for the squares $S_{k, l}$. By (3.21) the squares $S_{k, l}$ are contained in $S\left(F\left(w_{k}\right), \frac{1}{4} h\left(u_{k}\right)\right)$ and thus

$$
v_{k, l} \in V_{k, l} \subset W_{k} .
$$

We want to show that $S_{k, l}$ is admissible for at least one half of the indices $l$, provided $c_{0}$ is sufficiently large. In order to do so, we first note that (3.6) yields

$$
\delta^{2} \rho_{k}=\frac{\delta^{2}}{x^{p}} h^{\prime}\left(u_{k}\right) \geq \frac{\delta^{2}}{x^{p}} h^{\prime}\left(\frac{1}{2} x\right) \geq \frac{\delta^{2}}{x^{p}} \exp \left(\frac{1}{28} x\right)>100,
$$

if $c_{0}$ and hence $x$ is sufficiently large. Also, by (3.21),

$$
\delta^{2} \rho_{k}<\frac{1}{4} h\left(u_{k}\right)=\frac{1}{4} \operatorname{Re} F\left(w_{k}\right) .
$$


This means that each square $S_{k, l}$ has the size required to be admissible. Denoting by $I_{k}$ the set of all $l \in\left\{0,1, \ldots, m_{k}\right\}$ for which $S_{k, l}$ is admissible, we thus have

$$
I_{k}=\left\{l: \operatorname{length}\left(J_{k, l} \cap L\right) \geq \frac{7}{4} \delta^{2} \rho_{k}\right\} .
$$

With $I_{k}^{\prime}=\left\{0,1, \ldots, m_{k}\right\} \backslash I_{k}$ we obtain length $\left(J_{k, l} \backslash L\right)>\frac{1}{4} \delta^{2} \rho_{k}$ for $k \in I_{k}^{\prime}$. Now suppose that $\left|I_{k}\right|<\frac{1}{2}\left(m_{k}+1\right)$ so that $\left|I_{k}^{\prime}\right| \geq \frac{1}{2}\left(m_{k}+1\right)$. This implies that

$$
\begin{aligned}
\operatorname{length}\left(\left[\frac{3}{4} h\left(u_{k}\right), \frac{5}{4} h\left(u_{k}\right)\right] \backslash L\right) & \geq \sum_{l=0}^{m_{k}} \operatorname{length}\left(J_{k, l} \backslash L\right) \\
& \geq \sum_{l \in I_{k}^{\prime}} \operatorname{length}\left(J_{k, l} \backslash L\right) \\
& \geq\left|I_{k}^{\prime}\right| \frac{1}{4} \delta^{2} \rho_{k} \\
& \geq \frac{1}{8}\left(m_{k}+1\right) \delta^{2} \rho_{k} \\
& \geq \frac{1}{8} \delta r_{k} x^{p} \delta^{2} \rho_{k} \\
& =\frac{1}{8} \delta^{3} h\left(u_{k}\right) .
\end{aligned}
$$

Since $L$ has density 1 , this is a contradiction if $c_{0}$ and hence $u_{k}$ is sufficiently large. Thus

$$
\left|I_{k}\right| \geq \frac{1}{2}\left(m_{k}+1\right) \geq \frac{1}{2} \delta r_{k} x^{p} .
$$

As already mentioned, it follows from (3.21) that the squares $S_{k, l}$ are contained in $S\left(F\left(w_{k}\right), \frac{1}{4} h\left(u_{k}\right)\right)$ and thus, in particular,

$$
F\left(w_{k}\right)+l \delta \rho_{k} \in S\left(F\left(w_{k}\right), \frac{1}{4} h\left(u_{k}\right)\right) \subset B\left(F\left(w_{k}\right), \frac{1}{2} h\left(u_{k}\right)\right)
$$

for $0 \leq l \leq m_{k}$. Koebe's distortion theorem (2.3) now yields

$$
\frac{1}{81} \leq \frac{\left|\varphi_{k}^{\prime}\left(F\left(w_{k}\right)+l \delta \rho_{k}\right)\right|}{\left|\varphi_{k}^{\prime}\left(F\left(w_{k}\right)\right)\right|} \leq 81
$$

and hence, by Koebe's distortion theorem (2.2),

$$
V_{k, l} \subset B\left(v_{k, l}, 4\left|\varphi_{k}^{\prime}\left(F\left(w_{k}\right)+l \delta \rho_{k}\right)\right| \delta^{2} \rho_{k}\right) \subset B\left(v_{k, l}, 324\left|\varphi_{k}^{\prime}\left(F\left(w_{k}\right)\right)\right| \delta^{2} \rho_{k}\right) .
$$

Using (3.17) and the definition of $\rho_{k}$ in 3.20 we obtain

$$
V_{k, l} \subset B\left(v_{k, l}, \frac{324 \delta^{2}}{x^{p}}\right) .
$$

Similarly, it follows from Koebe's $\frac{1}{4}$-theorem (2.4) that

$$
V_{k, l} \supset B\left(v_{k, l}, \frac{\delta^{2}}{324 x^{p}}\right) .
$$

With $c_{1}=\delta^{2} / 324$ and $c_{2}=324 \delta^{2}$ we thus have

$$
B\left(v_{k, l}, \frac{c_{1}}{x^{p}}\right) \subset V_{k, l} \subset B\left(v_{k, l}, \frac{c_{2}}{x^{p}}\right) .
$$


Next we note that it follows from Lemma 2.3 together with (3.24) and (3.20) that

$$
\begin{aligned}
& \left|v_{k, l+1}-v_{k, l}-\frac{\delta}{x^{p}}\right| \\
= & \left|\varphi_{k}\left(F\left(w_{k}\right)+(l+1) \delta \rho_{k}\right)-\varphi_{k}\left(F\left(w_{k}\right)+l \delta \rho_{k}\right)-\varphi_{k}^{\prime}\left(F\left(w_{k}\right)\right) \delta \rho_{k}\right| \\
\leq & 26\left|\varphi_{k}^{\prime}\left(F\left(w_{k}\right)\right)\right| \frac{(l+1) \delta \rho_{k}}{h\left(u_{k}\right)} \delta \rho_{k} \\
= & \frac{26 \delta^{2}(l+1) \rho_{k}^{2}}{h^{\prime}\left(u_{k}\right) h\left(u_{k}\right)} \\
\leq & \frac{26 \delta^{3} r_{k} x^{p} \rho_{k}^{2}}{h^{\prime}\left(u_{k}\right) h\left(u_{k}\right)} \\
= & \frac{26 \delta^{3}}{x^{p}}
\end{aligned}
$$

for $0 \leq l \leq m_{k}-1$. It follows that

$$
\operatorname{Re} v_{k, l+1}-\operatorname{Re} v_{k, l} \geq \frac{\delta}{x^{p}}-\frac{26 \delta^{3}}{x^{p}}
$$

For sufficiently small $\delta$ we have $\delta-26 \delta^{3}>324 \tau \delta^{2}=\tau c_{2}$. Hence

$$
\operatorname{Re} v_{k, l+1}-\operatorname{Re} v_{k, l} \geq \frac{\tau c_{2}}{x^{p}} .
$$

If $k, k^{\prime} \in\{1,2, \ldots, n\}, k \neq k^{\prime}, l \in I_{k}$ and $l^{\prime} \in I_{k^{\prime}}$, then $\operatorname{Re} v_{k, l} \in\left(u_{k}-2 r_{k}, u_{k}+2 r_{k}\right)$ by (3.22) and (3.19). On the other hand, Re $v_{k^{\prime}, l^{\prime}} \notin\left(u_{k}-3 r_{k}, u_{k}+3 r_{k}\right)$ since, by (3.14),

$$
\left(u_{k}-3 r_{k}, u_{k}+3 r_{k}\right) \cap\left(u_{k^{\prime}}-3 r_{k^{\prime}}, u_{k^{\prime}}+3 r_{k^{\prime}}\right)=\emptyset .
$$

Since $u_{k} \in L^{\prime} \subset L$, it follows from (3.3) that

$$
\left|\operatorname{Re} v_{k, l}-\operatorname{Re} v_{k^{\prime}, l^{\prime}}\right| \geq r_{k}=\frac{h\left(u_{k}\right)}{h^{\prime}\left(u_{k}\right)} \geq \frac{1}{u_{k}^{p}} \geq\left(\frac{4}{5}\right)^{p} \frac{1}{x^{p}}
$$

and thus

$$
\left|\operatorname{Re} v_{k, l}-\operatorname{Re} v_{k^{\prime}, l^{\prime}}\right| \geq \frac{\tau c_{2}}{x^{p}}
$$

if $\delta$ and hence $c_{2}$ is sufficiently small.

We also note that it follows from Koebe's distortion theorem (2.2) together with (3.17), (3.20), (3.13) and (3.4) that

$$
\begin{aligned}
\left|v_{k, l}-w_{k}\right| & =\left|\varphi_{k}\left(F\left(w_{k}\right)+l \delta \rho_{k}\right)-\varphi_{k}\left(F\left(w_{k}\right)\right)\right| \\
& \leq\left|\varphi_{k}^{\prime}\left(F\left(w_{k}\right)\right)\right| 4 l \delta \rho_{k} \\
& \leq \frac{4 m_{k} \delta \rho_{k}}{h^{\prime}\left(u_{k}\right)} \\
& \leq \frac{4 \delta^{2} r_{k} x^{p} \rho_{k}}{h^{\prime}\left(u_{k}\right)} \\
& =4 \delta^{2} r_{k} \\
& \leq 16 \pi \delta^{2} .
\end{aligned}
$$

For small $\delta$ we thus have

$$
B\left(v_{k, l}, \frac{\tau c_{2}}{x^{p}}\right) \subset B\left(w_{k}, 1\right) .
$$


Recall that $\operatorname{Re} w_{k}=u_{k} \in L^{\prime}$ and so it follows from (3.12) and (3.16) that

$$
w_{k} \in\left\{\zeta \in \mathbb{C}:|\operatorname{Re} \zeta-\operatorname{Re} z| \leq \frac{1}{4} r-1,|\operatorname{Im} \zeta-\operatorname{Im} z| \leq \pi\right\}
$$

Thus

$$
B\left(v_{k, l}, \frac{\tau c_{2}}{x^{p}}\right) \subset\left\{\zeta \in \mathbb{C}:|\operatorname{Re} \zeta-\operatorname{Re} z| \leq \frac{1}{4} r,|\operatorname{Im} \zeta-\operatorname{Im} z| \leq \pi+1\right\} .
$$

Finally we note that it follows from (3.22) that

$$
F\left(v_{k, l}\right) \in F\left(V_{k, l}\right) \subset F\left(W_{k}\right)=S\left(F\left(w_{k}\right), \frac{1}{4} h\left(u_{k}\right)\right) .
$$

Also, since $u_{k} \in L^{\prime}$, it follows from (3.12) that

$$
\operatorname{Re} F\left(v_{k, l}\right) \geq \operatorname{Re} F\left(w_{k}\right)-\frac{1}{4} h\left(u_{k}\right)=\frac{3}{4} h\left(u_{k}\right) \geq \frac{3}{4} h\left(x-\frac{1}{4} r\right) \geq \frac{3}{4} h\left(\frac{7}{8} x\right) .
$$

Using (3.5) we obtain

$$
\operatorname{Re} F\left(v_{k, l}\right) \geq \exp \left(\frac{1}{15} x\right)
$$

if $c_{0}$ and hence $x$ is sufficiently large.

We now put

$$
X=\left\{v_{k, l}: k \in\{1,2, \ldots, n\}, l \in I_{k}\right\}
$$

Then

$$
m=|X|=\sum_{k=1}^{n}\left|I_{k}\right| \geq \frac{1}{2} \delta x^{p} \sum_{k=1}^{n} r_{k} \geq \frac{1}{240} \delta x^{p} r
$$

by (3.23) and (3.15). Thus $m \geq c_{3} x^{p} r$ for $c_{3}=\delta / 240$. By (3.26) and (3.27) we can write $X=\left\{a_{1}, a_{2}, \ldots, a_{m}\right\}$ with $\operatorname{Re} a_{1}<\operatorname{Re} a_{2}<\cdots<\operatorname{Re} a_{m}$ and, putting $A_{j}=V_{k, l}$ if $a_{j}=v_{k, l}$, we deduce from (3.25), (3.26), (3.27), (3.28) and (3.29) that, if $\delta$ is chosen to be sufficiently small, then (3.8), (3.9), (3.10) and (3.11) hold. Finally, it follows from the construction that $F$ maps $A_{j}$ bijectively onto an admissible square centred at $F\left(a_{j}\right)$, for $j=1,2, \ldots, m$.

\section{Proof of Theorem 1.2}

We now use Lemma 3.2 to construct the set $E_{p}$. Let $c_{0}$ be the constant obtained from Lemma 3.2 for fixed $\tau>1$. (The condition for $\tau$ will be specified later.) Let $Q_{0}$ be an admissible square $S\left(z_{0}, r_{0}\right)$ such that $\operatorname{Re} z_{0}=x_{0}>c_{0}$. For each $n \in \mathbb{N} \cup\{0\}$ we will define a finite collection $\mathcal{E}_{n}$ of compact, pairwise disjoint subsets of $Q_{0}$ with the following properties: for each $Q \in \mathcal{E}_{n}$, the set $F^{n}(Q)$ is an admissible square, each $Q \in \mathcal{E}_{n}$ contains at least one element of $\mathcal{E}_{n+1}$ and each $Q^{\prime} \in \mathcal{E}_{n+1}$ is contained in a unique $Q \in \mathcal{E}_{n}$.

We start by putting $\mathcal{E}_{0}=\left\{Q_{0}\right\}$. Now suppose that $\mathcal{E}_{n}$ has been defined and let $Q \in \mathcal{E}_{n}$. Let $A_{1}, \ldots, A_{m}$ be the sets obtained by applying Lemma 3.2 to the admissible square $S(z, r)=F^{n}(Q)$. For $k \in \mathbb{Z}$ and $j \in\{1, . ., m\}$, we put

$$
A_{j, k}=\left\{\zeta+2 \pi i k: \zeta \in A_{j}\right\} .
$$

Now let $\varphi$ be the branch of the inverse function of $F^{n}$ that maps $S(z, r)$ to $Q$. We define

$$
\mathcal{E}_{n+1}(Q)=\left\{\varphi\left(A_{j, k}\right): A_{j, k} \subset S\left(z, \frac{1}{4} r\right)\right\}
$$

and

$$
\mathcal{E}_{n+1}=\bigcup_{Q \in \mathcal{E}_{n}} \mathcal{E}_{n+1}(Q)
$$

Then $\mathcal{E}_{n+1}$ has the required properties. 
We define

$$
\overline{\mathcal{E}}_{n}=\bigcup_{Q \in \mathcal{E}_{n}} Q
$$

and

$$
E_{p}=\bigcap_{n=0}^{\infty} \overline{\mathcal{E}}_{n}
$$

It follows from the construction and (3.10) that, for each $z \in E_{p}$,

$$
\lim _{n \rightarrow \infty} \operatorname{Re} F^{n}(z)=\infty
$$

as required. In fact it follows that $\exp (z)$ belongs to the set $Z(f, D)$ defined in Section 1 .

We estimate the Hausdorff dimension of $E_{p}$ using the following result which is part of Frostman's Lemma; see, for example, [10, Proposition 4.9].

Lemma 4.1. Let $E$ be a compact subset of $\mathbb{C}$. Suppose that there exist a probability measure $\mu$ supported on $E$ and positive constants $c, \tilde{r}$ and $t$ such that, for each $z \in E$ and each $r \in(0, \tilde{r})$,

$$
\mu(B(z, r)) \leq c r^{t}
$$

Then $\operatorname{dim}(E) \geq t$.

Following [14] we construct a sequence of probability measures on $Q_{0}$. Let $\mu_{0}$ be the Lebesgue measure on $Q_{0}$ rescaled so that $\mu_{0}\left(Q_{0}\right)=1$. Then we construct the measure $\mu_{n}$ supported on $\overline{\mathcal{E}}_{n}$ inductively. Suppose that the measure $\mu_{n}$ on $\overline{\mathcal{E}}_{n}$ has been defined and let $Q_{n} \in \mathcal{E}_{n}$. The measure $\mu_{n+1}$ is defined as follows. If $A \subset \overline{\mathcal{E}}_{n+1} \cap Q_{n}$ then

$$
\mu_{n+1}(A)=\frac{\operatorname{area}\left(Q_{n}\right)}{\sum_{Q \in \mathcal{E}_{n+1}\left(Q_{n}\right)} \operatorname{area}(Q)} \mu_{n}(A)
$$

and, if $A \subset Q_{0} \backslash \overline{\mathcal{E}}_{n+1}$, then

Note that

$$
\mu_{n+1}(A)=0
$$

$$
\mu_{n+1}\left(\overline{\mathcal{E}}_{n+1} \cap Q_{n}\right)=\mu_{n}\left(Q_{n}\right)
$$

and that, for every $k \geq n$,

$$
\mu_{k}\left(Q_{n}\right)=\mu_{n}\left(Q_{n}\right)
$$

Thus there exists a unique measure $\mu$ supported on $E_{p}$ such that

$$
\mu\left(Q_{n}\right)=\mu_{n}\left(Q_{n}\right)
$$

for each set $Q_{n} \in \mathcal{E}_{n}$ and each $n \in \mathbb{N}$.

We now let $z \in E_{p}$. Our aim is to estimate $\mu(B(z, r))$ for $r$ sufficiently small. Let $Q_{n}(z)$ be the unique element of $\mathcal{E}_{n}$ that contains $z$. Then, for each $n \in \mathbb{N}$, we have $Q_{n}(z) \subset Q_{n-1}(z)$ and, by construction, $F^{n}\left(Q_{n}(z)\right)=S\left(z_{n}, r_{n}\right)$ for some admissible square $S\left(z_{n}, r_{n}\right)$.

Let $x_{n}=\operatorname{Re} z_{n}$. Then, by (3.10),

$$
x_{n} \geq \exp \left(\frac{1}{15} x_{n-1}\right) .
$$

We put

$$
d_{n}(z)=\operatorname{diam} Q_{n}(z)
$$

and denote the density of $\overline{\mathcal{E}}_{n+1}$ in $Q_{n}(z)$ by

$$
\Delta_{n}(z)=\frac{\sum_{Q \in \mathcal{E}_{n+1}\left(Q_{n}(z)\right)} \operatorname{area} Q}{\operatorname{area} Q_{n}(z)}
$$


We now estimate the quantities $\Delta_{n}(z)$ and $d_{n}(z)$. In order to do this, we first prove that there is a uniform bound for the distortion of $F^{n}$ on each set $Q \in \mathcal{E}_{n}$. (Recall that if a function $f$ is univalent on a set $S$ then the distortion of $f$ on $S$ is $\sup _{u, v \in S} \frac{\left|f^{\prime}(u)\right|}{\left|f^{\prime}(v)\right|}$. $)$

Lemma 4.2. There exists $K>0$ such that, if $n \in \mathbb{N}$ and $Q \in \mathcal{E}_{n}$ with $F^{n}(Q)=S\left(z^{\prime}, r^{\prime}\right)$, if $\varphi$ is the branch of $F^{-n}$ that maps $S\left(z^{\prime}, r^{\prime}\right)$ to $Q$ and if $\tilde{Q}=\varphi\left(B\left(z^{\prime}, \sqrt{2} r^{\prime}\right)\right)$, then

$$
\sup _{u, v \in \tilde{Q}} \frac{\left|\left(F^{n}\right)^{\prime}(u)\right|}{\left|\left(F^{n}\right)^{\prime}(v)\right|}<K \text {. }
$$

Proof. Since the branch of $F^{-1}$ that maps $F^{n}(Q)$ to $F^{n-1}(Q)$ is univalent in $B\left(z^{\prime}, 2 r^{\prime}\right)$, it follows from Koebe's distortion theorem (2.3) that the distortion of $F$ on $F^{n-1}(\tilde{Q})$ is bounded by the constant

$$
K_{1}=\frac{(\sqrt{2}+1)^{4}}{(\sqrt{2}-1)^{4}}
$$

Also, by construction, there is an admissible square $S\left(z^{\prime \prime}, r^{\prime \prime}\right)$ such that $F^{n-1}(Q) \subset$ $S\left(z^{\prime \prime}, \frac{1}{4} r^{\prime \prime}\right)$ and $F^{n-1}(\tilde{Q}) \subset B\left(z^{\prime \prime}, \frac{1}{2} r^{\prime \prime}\right)$. The branch of $F^{-(n-1)}$ that maps $F^{n-1}(\tilde{Q})$ onto $\tilde{Q}$ is univalent in $B\left(z^{\prime \prime}, r^{\prime \prime}\right)$ and so, by Koebe's distortion theorem (2.3), the distortion of $F^{n-1}$ on $\tilde{Q}$ is bounded by the constant $K_{2}=81$. The result now follows by putting $K=K_{1} K_{2}<10^{4}$.

We now use the result of Lemma 4.2 to obtain estimates for the density $\Delta_{n}(z)$ and the diameter $d_{n}(z)$.

Lemma 4.3. There exists a constant $c_{5}>0$ such that, for $n=0,1,2, \ldots$,

$$
\Delta_{n}(z) \geq \frac{c_{5}}{x_{n}^{p}}
$$

Proof. It follows from Lemma 4.2 that

$$
\Delta_{n}(z) \geq \frac{1}{K^{2}} \frac{\sum_{Q \in \mathcal{E}_{n+1}\left(Q_{n}(z)\right)} \operatorname{area} F^{n}(Q)}{\operatorname{area} F^{n}\left(Q_{n}(z)\right)} .
$$

By construction,

$$
F^{n}\left(\bigcup_{Q \in \mathcal{E}_{n+1}\left(Q_{n}(z)\right)} Q\right)=\bigcup_{A_{j, k} \subset S\left(z_{n}, r_{n} / 4\right)} A_{j, k},
$$

where $A_{j, k}=\left\{\zeta+2 \pi i k: \zeta \in A_{j}\right\}$ and $A_{j}$ is one of the sets obtained by applying Lemma 3.2 to $F^{n}\left(Q_{n}(z)\right)=S\left(z_{n}, r_{n}\right)$. Note that there are at least $c_{3} r_{n} x_{n}^{p}$ such sets $A_{j}$ and, by (3.8), each of these sets satisfies

$$
\text { area } A_{j} \geq \pi \frac{c_{1}^{2}}{x_{n}^{2 p}} .
$$

Also, for each $j$, the set $\left\{k: A_{j, k} \subset S\left(z_{n}, \frac{r_{n}}{4}\right)\right\}$ has at least $\frac{r_{n}}{4 \pi}-2$ elements. Since $r_{n}>100$,

$$
\frac{r_{n}}{4 \pi}-2>\frac{r_{n}}{8 \pi}
$$

and so

$$
\Delta_{n}(z) \geq \frac{c_{1}^{2} c_{3}}{32 K^{2}} \frac{1}{x_{n}^{p}} .
$$


Lemma 4.4. There exist constants $c_{6}, c_{7}>0$ such that, for $n=0,1,2 \ldots$ and for each $Q \in \mathcal{E}_{n+1}\left(Q_{n}(z)\right)$,

$$
\frac{c_{6}}{\left|\left(F^{n}\right)^{\prime}(z)\right| x_{n}^{p}} \leq \operatorname{diam} Q \leq \frac{c_{7}}{\left|\left(F^{n}\right)^{\prime}(z)\right| x_{n}^{p}}
$$

In particular, for $Q=Q_{n+1}(z)$ we have

$$
\frac{c_{6}}{\left|\left(F^{n}\right)^{\prime}(z)\right| x_{n}^{p}} \leq d_{n+1}(z) \leq \frac{c_{7}}{\left|\left(F^{n}\right)^{\prime}(z)\right| x_{n}^{p}}
$$

Proof. Since $F^{n}(Q)$ is one of the sets $A_{j, k}$ in $S\left(z_{n}, r_{n}\right)$, it follows from (3.8) that $F^{n}(Q)$ contains a ball of radius $c_{1} / x_{n}^{p}$ and is contained in a ball of radius $c_{2} / x_{n}^{p}$ which is contained in $S\left(z_{n}, r_{n}\right)$. Hence

$$
\frac{2 c_{1}}{x_{n}^{p}} \frac{1}{\sup _{u \in Q}\left|\left(F^{n}\right)^{\prime}(u)\right|} \leq \operatorname{diam} Q \leq \frac{2 c_{2}}{x_{n}^{p}} \frac{1}{\inf _{u \in Q}\left|\left(F^{n}\right)^{\prime}(u)\right|} .
$$

It now follows from Lemma 4.2 that

$$
\frac{2 c_{1}}{K} \frac{1}{x_{n}^{p}\left|\left(F^{n}\right)^{\prime}(z)\right|} \leq \operatorname{diam} Q \leq \frac{2 c_{2} K}{x_{n}^{p}\left|\left(F^{n}\right)^{\prime}(z)\right|} .
$$

We now obtain an estimate for the derivative $\left|\left(F^{n}\right)^{\prime}(z)\right|$ in terms of $x_{n}$.

Lemma 4.5. For each $\delta>0$, there exists $n_{0}>0$ such that, for $n>n_{0}$,

$$
\left|\left(F^{n}\right)^{\prime}(z)\right| \geq \frac{x_{n}}{8 \pi}
$$

Proof. It follows from Koebe's $\frac{1}{4}$-theorem (2.4) that if $\varphi$ is the branch of $F^{-1}$ that maps $F(z)$ to $z$ then

$$
\varphi(B(F(z), \operatorname{Re} F(z))) \supset B\left(z, \frac{\operatorname{Re} F(z)}{4 \mid\left(F^{\prime}(z) \mid\right.}\right) .
$$

Since $\varphi(B(F(z), \operatorname{Re} F(z)))$ contains no vertical segments of length $2 \pi$ we obtain

$$
\left|F^{\prime}(z)\right| \geq \frac{\operatorname{Re} F(z)}{4 \pi}
$$

As $\operatorname{Re} F^{i}(z)$ is much bigger than $4 \pi$ for $i=1, \ldots, n$ and $\operatorname{Re} F^{n}(z) \geq x_{n}-r_{n} \geq x_{n} / 2$, it follows that

$$
\left|\left(F^{n}\right)^{\prime}(z)\right| \geq \frac{\operatorname{Re} F^{n}(z)}{4 \pi} \geq \frac{x_{n}}{8 \pi}
$$

It follows from (4.4) and Lemma 4.5 that, for large $n$,

$$
d_{n+1}(z) \leq \frac{8 \pi c_{7}}{x_{n}^{p+1}}
$$

so $\lim _{n \rightarrow \infty} d_{n}(z)=0$ and

$$
\{z\}=\bigcap_{n=1}^{\infty} Q_{n}(z) .
$$

Since $Q_{n+1}(z) \subset Q_{n}(z)$ we have $d_{n+1}(z) \leq d_{n}(z)$. Thus, for $r$ sufficiently small, there exists a unique $n$ such that

$$
d_{n+1}(z) \leq r<d_{n}(z)
$$


Now fix $\delta \in(0,1)$. We may assume that $r<1$ is small enough to ensure that $n>n_{0}$, where $n_{0}$ is defined as in Lemma 4.5. Before we estimate the measure $\mu$ of $B(z, r)$ we shall show that, for $\tau$ sufficiently large, the ball $B(z, r)$ meets exactly one set in $\mathcal{E}_{n}$, namely the set $Q_{n}(z)$. We now fix $\tau>2 K+2$.

Lemma 4.6. For each $n \in \mathbb{N}$, if $Q, Q^{\prime} \in \mathcal{E}_{n}\left(Q_{n-1}(z)\right)$ then $\operatorname{dist}\left(Q, Q^{\prime}\right) \geq \operatorname{diam} Q$.

Proof. Let $Q, Q^{\prime} \in \mathcal{E}_{n}\left(Q_{n-1}(z)\right)$. It follows from Lemma 4.2 that

$$
\frac{\operatorname{diam} Q}{\operatorname{dist}\left(Q, Q^{\prime}\right)} \leq K \frac{\operatorname{diam} F^{n-1}(Q)}{\operatorname{dist}\left(F^{n-1}(Q), F^{n-1}\left(Q^{\prime}\right)\right)}
$$

It follows from the construction, (3.8) and (3.11) that

$$
\operatorname{diam} F^{n-1}(Q) \leq \frac{2 c_{2}}{x_{n-1}^{p}} \quad \text { and } \quad \operatorname{dist}\left(F^{n-1}(Q), F^{n-1}\left(Q^{\prime}\right)\right) \geq \frac{(\tau-2) c_{2}}{x_{n-1}^{p}}
$$

and so

$$
\frac{\operatorname{diam} Q}{\operatorname{dist}\left(Q, Q^{\prime}\right)} \leq \frac{2 K}{\tau-2}
$$

The result now follows since $\tau>2 K+2$.

Now let

$$
\mathcal{U}_{n}=\left\{Q \in \mathcal{E}_{n+1}\left(Q_{n}(z)\right): Q \cap B(z, r) \neq \emptyset\right\}
$$

Note that it follows from Lemma 3.2. Lemma 4.2 and (4.6) that, if $Q \in \mathcal{E}_{n}$ and $Q \cap B(z, r) \neq \emptyset$, then $Q=Q_{n}(z)$. So, by (4.6), (4.1), Lemma 4.3, and (4.3),

$$
\begin{aligned}
\mu(B(z, r)) & =\mu\left(B(z, r) \cap Q_{n}(z)\right) \\
& \leq \sum_{Q \in \mathcal{U}_{n}} \mu(Q) \\
& =\sum_{Q \in \mathcal{U}_{n}} \mu_{n+1}(Q) \\
& \leq \sum_{Q \in \mathcal{U}_{n}}\left(\prod_{j=0}^{n} \Delta_{j}(z)\right)^{-1} \frac{\operatorname{area} Q}{\operatorname{area} Q_{0}} \\
& \leq\left|\mathcal{U}_{n}\right| \frac{\left(x_{0} \ldots x_{n-1}\right)^{p}}{x_{n}^{p}\left|\left(F^{n}\right)^{\prime}(z)\right|^{2}} \frac{c_{7}^{2}}{c_{5}^{n+1} \text { area } Q_{0}} .
\end{aligned}
$$

If $n$ is sufficiently large, then

$$
\mu(B(z, r)) \leq\left|\mathcal{U}_{n}\right| \frac{x_{0}^{p}\left(x_{1} \ldots x_{n-2}\right)^{p+1} x_{n-1}^{p}}{x_{n}^{p}\left|\left(F^{n}\right)^{\prime}(z)\right|^{2}}
$$

and so, by (4.2),

$$
\mu(B(z, r)) \leq\left|\mathcal{U}_{n}\right| x_{n}^{-p}\left|\left(F^{n}\right)^{\prime}(z)\right|^{-2} x_{n-1}^{p+\delta}
$$

In order to get an upper bound for $\left|\mathcal{U}_{n}\right|$ it is sufficient to estimate the number of sets $A_{j, k}$ in $S\left(z_{n}, r_{n}\right)$ which meet $F^{n}(B(z, r))$. By Lemma 4.2,

$$
\operatorname{diam}\left(F^{n}(B(z, r)) \cap S\left(z_{n}, r_{n}\right)\right) \leq 2 K\left|\left(F^{n}\right)^{\prime}(z)\right| r .
$$

It follows from (3.8) and (4.8) that there are at most $K\left|\left(F^{n}\right)^{\prime}(z)\right| r x_{n}^{p} / c_{1}$ values of $j$ for which $F^{n}(B(z, r)) \cap A_{j, k} \neq \emptyset$ for some $k \in \mathbb{Z}$. Also, for each such $j$, the maximum number of values of $k$ for which $F^{n}(B(z, r)) \cap A_{j, k} \neq \emptyset$ is at most $\frac{K}{\pi}\left|\left(F^{n}\right)^{\prime}(z)\right| r+1$. 
Now we consider two cases.

Case 1:

$$
\frac{K}{\pi}\left|\left(F^{n}\right)^{\prime}(z)\right| r<1
$$

Then

and hence, by (4.7),

$$
\left|\mathcal{U}_{n}\right| \leq \frac{K}{c_{1}}\left|\left(F^{n}\right)^{\prime}(z)\right| r x_{n}^{p}
$$

$$
\mu(B(z, r)) \leq \frac{K}{c_{1}} r \frac{x_{n-1}^{p+\delta}}{\left|\left(F^{n}\right)^{\prime}(z)\right|} .
$$

It follows from Lemma 4.5 and (4.4) that

$$
\frac{1}{\left|\left(F^{n}\right)^{\prime}(z)\right|} \leq\left(\frac{(8 \pi)^{p}}{x_{n}^{p}\left|\left(F^{n}\right)^{\prime}(z)\right|}\right)^{1 /(p+1)} \leq\left(\frac{(8 \pi)^{p}}{c_{6}}\right)^{1 /(p+1)} d_{n+1}(z)^{1 /(p+1)}
$$

By (4.9), Lemma 4.5 and (4.2),

$$
r<\frac{\pi}{K\left|\left(F^{n}\right)^{\prime}(z)\right|} \leq \frac{8 \pi^{2}}{K x_{n}}<\frac{1}{x_{n-1}^{(p+\delta) / \delta}} .
$$

It follows from (4.10), (4.11), (4.12) and (4.6) that there exists a positive constant $c_{8}$ such that

$$
\mu(B(z, r)) \leq c_{8} r^{1+\frac{1}{p+1}-\delta}
$$

Case 2:

$$
\frac{K}{\pi}\left|\left(F^{n}\right)^{\prime}(z)\right| r \geq 1 .
$$

In this case, it follows from the discussion after (4.8) that

$$
\left|\mathcal{U}_{n}\right| \leq \frac{2 K^{2}}{\pi c_{1}}\left|\left(F^{n}\right)^{\prime}(z)\right|^{2} r^{2} x_{n}^{p}
$$

so, by (4.7),

$$
\mu(B(z, r)) \leq \frac{2 K^{2}}{\pi c_{1}} x_{n-1}^{p+\delta} r^{2}
$$

It follows from (4.6), (4.4) and Lemma 4.5 that

$$
r<d_{n}(z) \leq \frac{c_{7}}{x_{n-1}^{p}\left|\left(F^{n-1}\right)^{\prime}(z)\right|} \leq \frac{8 \pi c_{7}}{x_{n-1}^{p+1}}
$$

and hence

$$
x_{n-1}^{p+\delta} \leq\left(\frac{8 \pi c_{7}}{r}\right)^{\frac{p+\delta}{p+1}} .
$$

Thus there exists a positive constant $c_{9}$ such that

$$
\mu(B(z, r)) \leq c_{9} r^{1+\frac{1}{1+p}-\frac{\delta}{p+1}} .
$$

In both cases, since $r<1$, we have

$$
\mu(B(z, r)) \leq \max \left\{c_{8}, c_{9}\right\} r^{1+\frac{1}{1+p}-\delta}
$$


so, by Lemma 4.1, for each $\delta \in(0,1)$,

$$
\operatorname{dim}\left(E_{p}\right) \geq 1+\frac{1}{1+p}-\delta
$$

Letting $\delta$ tend to 0 we obtain that

$$
\operatorname{dim}\left(E_{p}\right) \geq 1+\frac{1}{1+p}
$$

This completes the proof of Theorem 1.2 .

\section{Concluding Remarks}

1. The Hausdorff dimension of the set $E_{p}$ constructed in the proof of Theorem 1.2 is in fact equal to $1+1 /(p+1)$. To see this, consider the cover of $E_{p}$ by the sets in $\mathcal{E}_{n}$. Let $Q_{n} \in \mathcal{E}_{n}$. Then there exists an admissible square $S\left(z^{\prime}, r^{\prime}\right)$ with $r^{\prime}<\frac{1}{2} x=\frac{1}{2} \operatorname{Re} z^{\prime}$ and $F^{n}\left(Q_{n}\right)=S\left(z^{\prime}, r^{\prime}\right)$. It follows from Lemma 4.2 and Lemma 3.2 that, for each $s>1$,

$$
\begin{aligned}
\frac{\sum_{Q \in \mathcal{E}_{n+1}\left(Q_{n}\right)}(\operatorname{diam} Q)^{s}}{\left(\operatorname{diam} Q_{n}\right)^{s}} & \leq K^{s} \frac{\sum_{Q \in \mathcal{E}_{n+1}\left(Q_{n}\right)}\left(\operatorname{diam} F^{n}(Q)\right)^{s}}{\left(\operatorname{diam} F^{n}\left(Q_{n}\right)\right)^{s}} \\
& \leq \frac{K^{s}}{\left(2 \sqrt{2} r^{\prime}\right)^{s}} \frac{r^{\prime}}{\pi} \frac{r^{\prime} x^{p}}{c_{1}}\left(\frac{2 c_{2}}{x^{p}}\right)^{s} \\
& \leq c r^{(2-s)} x^{p(1-s)} \\
& <c x^{2-s+p(1-s)}
\end{aligned}
$$

where $c>0$ is a constant that is independent of $n \in \mathbb{N}$ and of the choice of $Q_{n} \in \mathcal{E}_{n}$. Now suppose that $s=1+\frac{1}{1+p}+\delta$, for some $\delta>0$. Then

$$
2-s+p(1-s)=1-\delta-\frac{1}{1+p}-\frac{p}{1+p}-p \delta=-\delta(1+p) .
$$

Thus, if $n$ and hence $x$ is sufficiently large,

$$
\frac{\sum_{Q \in \mathcal{E}_{n+1}\left(Q_{n}\right)}(\operatorname{diam} Q)^{s}}{\left(\operatorname{diam} Q_{n}\right)^{s}}<1 .
$$

Since $\max \left\{\operatorname{diam} Q: Q \in \mathcal{E}_{n}\right\} \rightarrow 0$ as $n \rightarrow \infty$, it follows that $\operatorname{dim} E_{p} \leq s$. The result now follows by letting $\delta \rightarrow 0$.

2. The examples in [23] of entire functions in the class $B$ which show that the estimate in Theorem 1.1 is sharp for $q>1$ have a logarithmic tract similar to the region

$$
\Omega=\left\{x+i y: x>1, y>\frac{x}{(\log x)^{q-1}}\right\} .
$$

The region $\Omega$ also appears in [12] where it is shown that, for $E_{\lambda}(z)=\lambda e^{z}$, the set of $z \in I\left(E_{\lambda}\right)$ for which $E_{\lambda}^{n}(z) \in \Omega$ for large $n$ has Hausdorff dimension $1+1 / q$.

3. Rempe [17] has recently shown that, if $f, g \in B$ and there exist quasiconformal homeomorphisms $\phi, \psi: \mathbb{C} \rightarrow \mathbb{C}$ such that $\phi \circ f=g \circ \psi$, then there exists $R>0$ and a quasiconformal homeomorphism $\theta: \mathbb{C} \rightarrow \mathbb{C}$ such that $\theta(f(z))=g(\theta(z))$ if $\left|f^{n}(z)\right| \geq R$ for all $n \geq 0$. Since quasiconformal homeomorphisms map sets of Hausdorff dimension 2 to sets of Hausdorff dimension 2, this implies that $\operatorname{dim} I(f)=2$ if $\operatorname{dim} I(g)=2$. Choosing $g=\lambda f$ with sufficiently small $\lambda$ we see that, in order to prove that $\operatorname{dim} I(f)=2$ for all functions of finite order in the class $B$, it is sufficient to consider such functions for which the Fatou set consists of a single attracting basin. 
Note that this kind of reasoning does not extend to the case where the dimension is less than 2, since then the Hausdorff dimension is not preserved by a quasiconformal homeomorphism. The sharp bounds for the distortion of Hausdorff dimension under quasiconformal mappings are given by a famous result of Astala [1].

In general, it is open as to whether two quasiconformally equivalent functions $f$ and $g$ can have escaping sets of different Hausdorff dimensions. It is known, however, that this cannot happen when the maps $\phi$ and $\psi$ can be chosen to be conformal.

\section{REFERENCES}

[1] K. Astala, Area distortion of quasiconformal mappings. Acta Math. 173 (1994) 37-60.

[2] K. Barański, Hausdorf dimension of hairs and ends for entire maps of finite order. Math. Proc. Cambridge Philos. Soc. 2008, doi:10.1017/S0305004108001515.

[3] K. Barański, B. Karpińska and A. Zdunik, Hyperbolic dimension of Julia sets of meromorphic maps with logarithmic tracts. Preprint, arXiv: 0711.2672.

[4] W. Bergweiler, Maximum modulus, characteristic, and area on the sphere. Analysis 10 (1990), 163-176; Erratum: 12 (1992), 67-69.

[5] W. Bergweiler, Iteration of meromorphic functions. Bull. Amer. Math. Soc. (N. S.) 29 (1993), 151188.

[6] W. Bergweiler and A. Eremenko, On the singularities of the inverse to a meromorphic function of finite order. Rev. Mat. Iberoamericana 11 (1995), 355-373.

[7] W. Bergweiler, P. J. Rippon and G. M. Stallard, Dynamics of meromorphic functions with direct or logarithmic singularities. Proc. London Math. Soc. 2008, doi:10.1112/plms/pdn007.

[8] A. E. Eremenko, On the iteration of entire functions, in Dynamical systems and ergodic theory. Banach Center Publications 23, Polish Scientific Publishers, Warsaw 1989, 339-345.

[9] A. E. Eremenko and M. Yu. Lyubich, Dynamical properties of some classes of entire functions. Ann. Inst. Fourier 42 (1992), 989-1020.

[10] K. Falconer, Fractal geometry. John Wiley \& Sons, Chichester, 2003.

[11] G. Jank and L. Volkmann, Einführung in die Theorie der ganzen und meromorphen Funktionen mit Anwendungen auf Differentialgleichungen. Birkhäuser, Basel, Boston, Stuttgart, 1985.

[12] B. Karpińska and M. Urbański, How points escape to infinity under exponential maps. J. London Math. Soc. (2) 73 (2006), 141-156.

[13] J. K. Langley, On the multiple points of certain meromorphic functions. Proc. Amer. Math. Soc. 123 (1995), 355-373.

[14] C. McMullen, Area and Hausdorff dimension of Julia sets of entire functions. Trans. Amer. Math. Soc. 300 (1987), 329-342.

[15] G. Pólya and G. Szegö, Problems and Theorems in Analysis I (Part III, problems 158-160). Springer, New York, 1972.

[16] L. Rempe, Siegel disks and periodic rays of entire functions. J. Reine Angew. Math., to appear.

[17] L. Rempe, Rigidity of escaping dynamics for transcendental entire functions. arXiv: math/0605058.

[18] P. J. Rippon and G. M. Stallard, Dimensions of Julia sets of meromorphic functions. J. London Math. Soc., (2) 71 (2005), 669-683.

[19] G. Rottenfußer, J. Rückert, L. Rempe and D. Schleicher, Dynamic rays of bounded-type entire functions. Stony Brook preprint ims07-05, arXiv: 0704.3213.

[20] H. Schubert, Über die Hausdorff-Dimension der Juliamenge von Funktionen endlicher Ordnung. Dissertation, University of Kiel, 2007.

[21] G. M. Stallard, The Hausdorff dimension of Julia sets of entire functions. Ergodic Theory Dynam. Systems 11 (1991), 769-777.

[22] G. M. Stallard, The Hausdorff dimension of Julia sets of entire functions II. Math. Proc. Cambridge Philos. Soc. 119 (1996), 513-536.

[23] G. M. Stallard, The Hausdorff dimension of Julia sets of entire functions IV. J. London Math. Soc. (2) 61 (2000), 471-488.

[24] M. Taniguchi, Size of the Julia set of structurally finite transcendental entire function. Math. Proc. Cambridge Philos. Soc. 135 (2003), 181-192. 
Mathematisches Seminar, Christian-Albrechts-Universität Zu Kiel, Ludewig-MeynStr. 4, D-24098 Kiel, Germany

E-mail address: bergweiler@math.uni-kiel.de

Faculty of Mathematics and Information Science, Warsaw University of Technology, Pl. Politechniki 1, 00-661 Warszawa, Poland

E-mail address: bkarpin@mini.pw.edu.pl

Department of Mathematics and Statistics, The Open University, Walton Hall, MilTON Keynes MK7 6AA, United Kingdom

E-mail address: g.m.stallard@open.ac.uk 